\title{
Diseño e implementación del plan de mantenimiento preventivo de los equipos de la empresa Granitos y Mármoles Acabados SAS
}

| Fecha de recibido: 17 de marzo del 2021 | Fecha de aprobación: 27 de septiembre del 2021 |

\section{María Gabriela Mago Ramos \\ Doctora en Ingeniería}

Docente, Universidad Libre Colombia

Rol de la investigadora: teórico, experimental y escritura https://orcid.org/0000-0001-7250-111X

$\triangle$ mariag.magor@unilibre.edu.co
Sebastián Rocha Pachón

Ingeniero Mecánico

Universidad Libre Colombia

Rol del investigador: teórico, experimental y escritura https://orcid.org/0000-0002-7142-0796

$\bowtie$ Sebastianrocha739@gmail.com

Cómo citar este artículo: Mago-Ramos, M., \& Rocha-Pachón, S. (2021). Diseño e implementación del plan de mantenimiento preventivo de los equipos de la empresa Granitos y Mármoles Acabados SAS. Ciencia y Poder Aéreo, 16(2), 98-111. https://doi.org/10.18667/cienciaypoderaereo.703 


\section{Diseño e implementación del plan de mantenimiento preventivo de los equipos de la empresa Granitos y Mármoles Acabados SAS}

Resumen: Esta investigación propone el diseño y la implementación del plan de mantenimiento preventivo en pequeñas industrias que empezaron en forma de proyectos familiares o de manera artesanal, las cuales no tuvieron la posibilidad de adoptar una estrategia de mantenimiento como el Mantenimiento Centrado en Confiabilidad (RCM), que permite identificar los equipos más críticos, mediante un análisis de criticidad para el proceso y adoptar estrategias a fin de posibilitar la eliminación de la ocurrencia de fallas. La metodología empleada en esta investigación, que fue aprendida en los cursos de Mantenimiento del programa de Ingeniería Mecánica de la Universidad Libre, permitió generar formatos que facilitaron el manejo de los equipos y la visualización de la información, así como otros documentos para el seguimiento y control de las actividades relacionadas con cada activo. La microempresa, caso de estudio, cuya razón social se denomina Granitos y Mármoles Acabados SAS presta servicios de mantenimiento a todo tipo de piedra natural hace más de 20 años, sin efectuar ninguna estrategia de mantenimiento para sus equipos, por lo que, buscando optimizar métodos productivos contempló la necesidad de implementar la estrategia. El estudio se complementó con un análisis financiero: cálculo de ROI y TIR, que posibilitó la evaluación de la inversión en mantenimiento, no solamente como un gasto. La aplicación de procedimientos teóricos y analíticos permitió verificar que los servicios que presta la empresa tienden a volverse más eficientes, demostrando con cifras la retribución económica que genera un plan de mantenimiento para este sector en específico. El aporte de esta investigación a las investigaciones futuras radica en la elaboración de un plan de mantenimiento en empresas PYME que no han tenido esta cultura técnica u operacional con los equipos, y que a largo plazo les genera un menor lucro cesante y una mayor productividad cuando se invierte en mantenimiento.

Palabras clave: diseño; implementación; plan de mantenimiento preventivo; equipos.

Abstract: This research proposes the design and implementation of a preventive maintenance plan for small industries that began in the form of family businesses, which have not had the chance of adopting a maintenance strategy such as Reliability Centered Maintenance (RCM). The proposal allows identifying the most critical equipment (through a criticality analysis) for the process in order to adopt strategies that eliminate failures. The methodology used in this research, learned in the maintenance courses of the Mechanical Engineering Program at Universidad Libre, allowed generating formats that facilitated the management of equipment and the visualization of information and other documents required for the monitoring and control of the activities related to each asset. The case study company, named Granitos y Mármoles Acabados SAS, is a micro-enterprise in the business of maintenance services to all types of natural stone, which has operated for over 20 years without carrying out any maintenance strategy for its equipment. This company considered the need to implement a maintenance strategy in order to optimize its production methods. The study was complemented with a financial analysis (ROI and IRR calculation) that allowed evaluating the investment in maintenance not only as an expense. By applying theoretical and analytical procedures, it was verified that the services provided by this company tend to become more efficient, proving with figures the economic retribution generated by a maintenance plan. The contribution of this research to future studies lies in the development of a maintenance plan for SMEs that have not implemented this technical or operational culture with their equipment, and that, in the long term, generates a decreased loss of profit and greater productivity.

Keywords: design; implementation; preventive maintenance plan; equipment.

Resumo: Esta pesquisa propõe a concepção e implementação do plano de manutenção preventiva em pequenas indústrias que se iniciaram na forma de projetos familiares ou de forma tradicional, que não tinham a possibilidade de adotar uma estratégia de manutenção como a Manutenção Centrada em Confiabilidade (RCM), que permite identificar os equipamentos mais críticos, através de uma análise de criticidade, para o processo e adotar estratégias que possibilitem a eliminação da ocorrência de falhas. A metodologia utilizada nesta pesquisa, que foi aprendida nos cursos de Manutenção do Programa de Engenharia Mecânica da Universidade Livre, permitiu gerar formatos que facilitam a gestão dos equipamentos e a visualização das informações, bem como outros documentos para o monitoramento e controle das atividades relacionadas a cada ativo. A empresa, caso de estudo, cuja denominação social se denomina "Granitos y Mármoles Acabados SAS" é uma microempresa que há mais de 20 anos presta serviços de manutenção a todo o tipo de pedra natural, sem levar a cabo qualquer estratégia de manutenção dos seus equipamentos, por isso para otimizar os métodos de produção, considerou a necessidade de implementá-la. O estudo foi complementado com uma análise financeira: cálculo do ROI e da TIR, que possibilitou avaliar o investimento em manutenção, não apenas como despesa. A aplicação de procedimentos teóricos e analíticos permitiu verificar que os serviços prestados pela empresa tendem a se tornar mais eficientes, demonstrando com números a retribuição econômica gerada por um plano de manutenção para este setor específico. A contribuição desta pesquisa para pesquisas futuras está no desenvolvimento de um plano de manutenção em empresas de pequeno e médio porte que não possuíam essa cultura técnica ou operacional com os equipamentos, e que no longo prazo gera menores lucros cessantes e maior produtividade quando é investido em manutenção.

Palavras-chave: design; implementação; plano de manutenção preventiva; equipes. 
El mantenimiento de los equipos es necesario realizar la función para la que son diseñados; es así que desde el inicio de la industrialización se ha concebido el mantenimiento como parte esencial del buen desempeño de las máquinas. Con los avances tecnológicos han surgido distintas maneras de gestionar las actividades destinadas a esto, todas ellas buscan la forma más eficaz y eficiente de asegurar que realicen una función deseada dentro de los parámetros establecidos, mediante la implementación de herramientas y estrategias que faciliten el análisis y la toma de decisiones. El Mantenimiento Centrado en Confiabilidad (RCM) es una de estas herramientas, la cual ha tenido gran acogida gracias a que, además de que resulta sencilla de implementar, brinda una manera de organizar los activos conociéndolos en forma más detallada. La implementación de esta estrategia es dinámica y depende del entorno o del contexto operacional (Lucía, 1990). Con este plan se muestra que las industrias que empiezan como proyectos familiares o de manera artesanal, y que no tuvieron la posibilidad de conocer dichas herramientas, pueden adoptar una estrategia de mantenimiento como el RCM, ya que les brinda una forma efectiva de organizar sus activos por medio de un procedimiento que permite conocer cada una de las fallas, y así tomar decisiones para mitigar el efecto. Durante este proceso se generan formatos que facilitan el manejo de los equipos y la visualización de la información, de igual forma sucede con los documentos necesarios para el seguimiento y control de las actividades relacionadas de cada activo.

Este es el caso de Granitos y Mármoles Acabados SAS, una microempresa que presta servicios de distribución, procesamiento y mantenimiento de todo tipo de piedra natural como mármoles o granitos en pisos o fachadas, donde se ha venido trabajando sin implementar ninguna estrategia de mantenimiento, lo que ha repercutido negativamente en el estado de los equipos y en el cumplimiento de metas, por lo que se contempla la necesidad de implementar esta estrategia para optimizar la empresa. El estudio se complementa con un análisis financiero que permite clarificar y evaluar que la inversión en mantenimiento no es solamente gasto. Además de determinar el retorno de la inversión (ROI), correspondiente al proyecto, se demuestra con cifras la retribución económica que genera un plan de mantenimiento para este sector.

\section{La empresa y sus procesos}

Granitos y Mármoles Acabados SAS es una microempresa del sector de construcción, creada en 1995; ha crecido a medida que el sector se ha desarrollado en Colombia, lo que le ha permitido adquirir experiencia y adaptarse a las nuevas tecnologías para prestar de manera eficaz y eficiente sus servicios. Cuenta con 10 empleados y 12 equipos; sus procesos han sido desarrollados de manera empírica y modificados a través de la experiencia propia, buscando optimizar los recursos. Esta empresa nunca ha tenido la oportunidad de aplicar estrategias o planes de mantenimiento, pero con motivo de esta investigación, se convertirá en pionera de su sector y así servirá como piloto para aplicaciones a empresas similares.

A continuación, en la figura 1 se muestra el diagrama de flujo para el proceso de pulido de áreas en piedra natural o mármol donde se evidencia el uso que se les da a los equipos susceptibles de mantenimiento, cuyas actividades son: destronque y pulido, acabado y brillado.

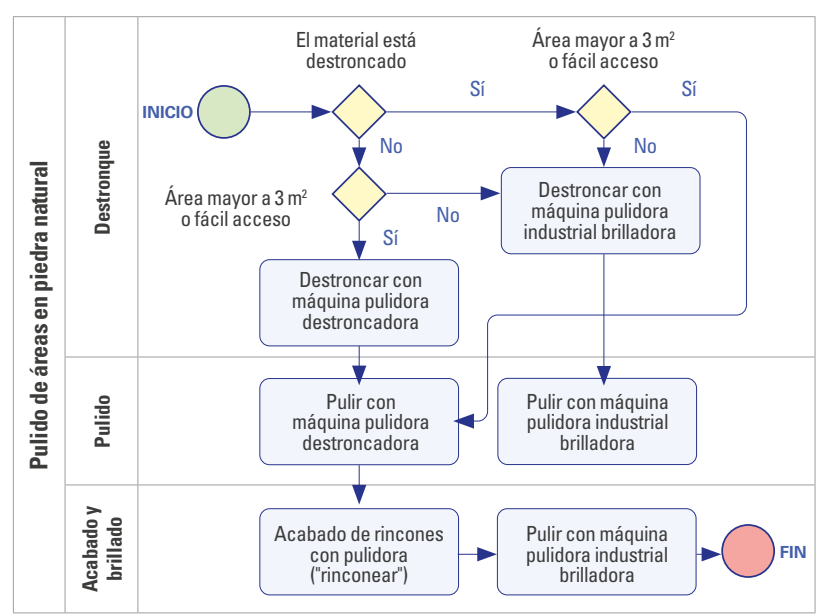

Figura 1. Flujograma del proceso empresa Granitos y Mármoles Acabados SAS

Fuente: elaboración propia. 
Destronque y pulido: desbaste hasta conseguir una superficie uniforme; esto se consigue utilizando una máquina pulidora-destroncadora la cual utiliza discos con incrustaciones de diamante industrial de granulometría n. ${ }^{\circ}$ 24-60-120; con la superficie uniforme se cambia a los discos de pulido, los cuales tienen una granulometría n. ${ }^{\circ}$ 220-400-600-800; en ocasiones estos procesos se deben realizar con una máquina pulidora industrial brilladora debido a que no todas las superficies son de fácil acceso.

Acabado y brillado: se aprovecha una reacción química entre el carbonato de calcio presente en el mármol y un cristalizante que satura la superficie y logra dar un brillo natural. La máquina pulidora industrial también es usada para esta tarea cambiando los discos de pulir por unos paños a los cuales se les pone lana de acero para, después, aplicar los cristalizantes uniformemente sobre la superficie.

\section{Descripción del problema de investigación}

En la industria hay empresas que tienen equipos susceptibles de mantenimiento, los cuales deben estar dentro de ciertos parámetros mínimos de operación, productividad y eficiencia; adicionalmente, deben cumplir con toda la normatividad de seguridad industrial exigida por algunas compañías, como manuales, fichas técnicas, procedimientos, planes de mantenimiento, certificados de reparación, entre otros. Existen micro o medianas empresas que no tienen implementada ninguna estrategia o metodología de mantenimiento, bien sea por su desconocimiento o porque desarrollan sus actividades de manera artesanal; debido a esto, se creó la necesidad de implementar planes de mantenimiento para dichos equipos con el fin de que estas empresas tengan un mejor control de los activos, optimizando los recursos y el tiempo para que se puedan ajustar a los lineamientos exigidos.

La implementación de un plan de mantenimiento busca principalmente los siguientes objetivos: mejorar la disponibilidad del equipo, disminuir los costos de mantenimiento y optimizar los recursos humanos (Gómez 2006; Mora, 2019). Hay diferentes estrategias que sirven como herramienta para el análisis de la información y su correcta implementación, la cual se debe seleccionar teniendo en cuenta el modo de operación, como también los procesos, activos, recursos financieros, personal calificado y tiempo de servicio. Con esto se establecerán estrategias que permitan el desarrollo de actividades enmarcadas hacia el logro de objetivos estratégicos operacionales y financieros. Los planes y programas de mantenimiento en sectores productivos traen ventajas a la operación y la calidad mediante el control de actividades y gestión de los recursos, lo cual permite establecer indicadores de fiabilidad que garanticen la disponibilidad de los activos. Esta premisa puede aplicarse también a las PYME que hacen parte de estos sectores, y más aún, cuando no tienen en sus procesos planes de mantenimiento, lo cual puede dar origen a varios interrogantes: ¿Cómo los planes de mantenimiento pueden mejorar la respuesta en los tiempos de servicio, favoreciendo costos operacionales y atención a los clientes?, y otra no menos importante (Villareal, 2017), ¿cómo se hacen más competitivas y con mayores oportunidades de negocio estas PYME, mediante la aplicación de buenas prácticas de mantenimiento?

\section{Justificación de la investigación}

Granitos y Mármoles Acabados SAS empezó como un proyecto familiar, que viene prestando sus servicios de mantenimiento de pisos y fachadas de una manera empírica, donde no hay una taxonomía definida para: los equipos, fichas técnicas, hojas de vida, procedimientos, listado de repuestos, plan de mantenimiento o registro; debido a esto, no se logra la premisa principal de un plan de mantenimiento ni se cumplen con los requisitos exigidos por compañías que implementan sistemas integrados de gestión, estas compañías exigen en sus procesos de selección que los equipos cumplan con las normas de seguridad industrial establecidas para celebrar contratos con ellas. En esta 
investigación se realizó un análisis del estado de los equipos de la empresa utilizando técnicas de recolección de información, con los indicadores necesarios para diseñar un plan de mantenimiento enfocado en la integridad de los activos, en pro de reducir costos y ser más competitivos en el mercado (Monsalve \& Tena, 2018).

\section{Metodología}

Esta investigación tiene un enfoque cualitativo, ya que para lograr su objetivo recolectó información basada en la observación y en principios no cuantificables ( $\mathrm{Da}$ Costa et al., 2010). El proyecto fue estructurado en una serie de fases metodológicas que se muestran en la figura 2.

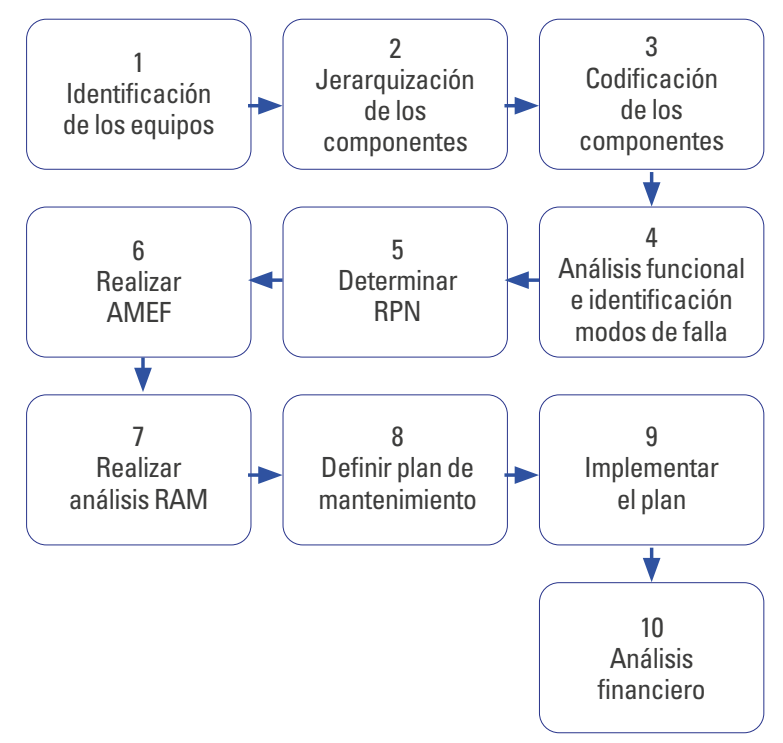

Figura 2. Metodología del proyecto

Fuente: elaboración propia.

\section{Identificación de equipos}

Se realizó un análisis de criticidad con el cual se priorizaron los equipos incluidos en el listado de equipos mantenibles, identificándolos mediante formatos de fichas de equipos, y en la creación de las hojas de vida.

\section{Jerarquización de los componentes}

Cada equipo se desglosó en sus sistemas y subsistemas hasta llegar a los componentes identificando las partes mantenibles, todo esto en un orden jerárquico.

\section{Codificación de los componentes}

Se creó un código único con el fin de facilitar la identificación, localización y manejo de los activos y sus componentes asociados.

Análisis funcional e identificación modos de falla

Para conocer e identificar los modos de falla se hizo un análisis funcional donde fue considerada cada función revelando las fallas funcionales; de esta manera, se identificaron los modos, así como el mecanismo de falla.

\section{Determinar RPN}

Se calculó el número ponderado de riesgo (RPN) según el nivel de severidad, ocurrencia y detección.

\section{Realizar AMEF}

En este formato se consigna de forma ordenada la información de los modos de falla, lo que facilita su interpretación y permite definir acciones correctivas para cada uno de los modos.

\section{Definir plan de mantenimiento}

Con dichas acciones se procedió a planificar el mantenimiento por medio de formatos de rutas de trabajo, las cuales organizaron eficientemente los recursos técnicos, humanos y financieros.

\section{Implementar el plan}

Las rutas fueron implementadas mediante un formato de órdenes de trabajo donde se incluyeron especificaciones claras de las tareas al operario, así mismo, con 
este documento se puede obtener un mejor control sobre la ejecución del plan.

\section{Análisis financiero}

Finalmente, se efectuó un análisis financiero con el fin de conocer los costos que se requerían para la implementación del plan, se evaluó la viabilidad del proyecto con el cálculo del ROI.

\section{Resultados}

En el diagrama de flujo del proceso que se presenta en la figura 1 para la sección de pulido en áreas de piedra natural se evidencia que se utilizan básicamente tres tipos de máquinas susceptibles de mantenimiento: máquina destroncadora-pulidora, máquina pulidora industrial brilladora y pulidoras, las cuales son utilizadas simultáneamente, pues la empresa cuenta con siete equipos en total; estos dos primeros son los equipos más antiguos, los cuales han sido reparados por los operarios de forma artesanal, debido a esto, la frecuencia de falla es alta-media, además las fallas de presentadas tienen un gran impacto en la producción (Salguero, 2010; Páramo, 2016). A continuación, se muestra en la tabla 1 la lista de equipos de mayor importancia para el proceso que también fueron evaluadas mediante un análisis de criticidad tal y como se muestra posteriormente en la tabla 2.

Tabla 1

Lista de equipos

\begin{tabular}{l|l|c|c|c|c}
\hline \multirow{2}{*}{ Empresa: } & Granitos y Mármoles Acabados SAS & Fecha & 02 & 09 & 2018 \\
\hline \multirow{2}{*}{ Área } & Equipo & \multicolumn{3}{|c}{ Sistema } \\
\cline { 3 - 6 } & Máquina destroncadora-pulidora 1 & ELE & MEC & HID \\
\cline { 2 - 6 } Destronque & Máquina destroncadora-pulidora 2 & $\mathrm{x}$ & $\mathrm{x}$ & $\mathrm{x}$ \\
\cline { 2 - 6 } & Máquina destroncadora-pulidora 3 & $\mathrm{x}$ & $\mathrm{x}$ & $\mathrm{x}$ \\
\hline Pulido & Máquina pulidora industrial brilladora 1 & $\mathrm{x}$ & $\mathrm{x}$ & $\mathrm{x}$ \\
\hline $\begin{array}{l}\text { Brillado y } \\
\text { acabdo }\end{array}$ & Máquina pulidora industrial brilladora 2 & $\mathrm{x}$ & $\mathrm{x}$ & \\
\hline
\end{tabular}

Fuente: elaboración propia.
Tabla 2

Análisis de criticidad

\begin{tabular}{|c|c|c|c|c|c|c|c|}
\hline \multirow{2}{*}{ Activo } & \multicolumn{5}{|c|}{ Impacto } & \multirow{2}{*}{ 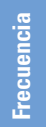 } & \multirow{2}{*}{$\begin{array}{l}\text { 롫 } \\
\text { 률 } \\
\text { 롱 }\end{array}$} \\
\hline & IPR & IP & IA & 10 & Total & & \\
\hline Máquina destroncadora-pulidora 1 & 4 & 2 & 1 & 3 & 10 & 4 & 40 \\
\hline Máquina destroncadora-pulidora 2 & 4 & 2 & 1 & 3 & 10 & 4 & 40 \\
\hline Máquina destroncadora-pulidora 3 & 2 & 2 & 1 & 3 & 8 & 4 & 32 \\
\hline Máquina industrial brilladora 1 & 3 & 1 & 1 & 2 & 7 & 3 & 21 \\
\hline Máquina industrial brilladora 2 & 3 & 1 & 1 & 2 & 7 & 3 & 21 \\
\hline Pulidora manual & 1 & 1 & 1 & 1 & 4 & 2 & 8 \\
\hline
\end{tabular}

\begin{tabular}{|c|c|c|c|c|c|c|c|c|c|c|c|c|c|c|c|}
\hline \multicolumn{16}{|c|}{$\begin{array}{l}\text { Matriz de criticidad } \\
\text { Consecuencia }\end{array}$} \\
\hline \multirow{5}{*}{ 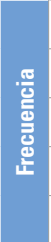 } & & 3 & 4 & 5 & 6 & 7 & 8 & 9 & 10 & 11 & 12 & 13 & 14 & 15 & 16 \\
\hline & 4 & 12 & 16 & 20 & 24 & 28 & 32 & 36 & 40 & 44 & 48 & 52 & 56 & 60 & 64 \\
\hline & 3 & 9 & 12 & 15 & 18 & 21 & 24 & 27 & 30 & 33 & 36 & 39 & 42 & 45 & 48 \\
\hline & 2 & 6 & 8 & 10 & 12 & 14 & 16 & 18 & 20 & 22 & 24 & 26 & 28 & 30 & 32 \\
\hline & 1 & 3 & 4 & 5 & 6 & 7 & 8 & 9 & 10 & 11 & 12 & 13 & 14 & 15 & 16 \\
\hline \multicolumn{5}{|c|}{ alta (crítico) } & & \multicolumn{5}{|c|}{$29 \leq$ Criticidad $\leq 64$} & & & & & \\
\hline \multicolumn{5}{|c|}{ media (vital) } & & \multicolumn{5}{|c|}{$15 \leq$ Criticidad $\leq 28$} & & & & & \\
\hline \multicolumn{5}{|c|}{ Baja (secundario) } & & \multicolumn{5}{|c|}{$29 \leq$ Criticidad $\leq 14$} & & & & & \\
\hline
\end{tabular}

Fuente: elaboración propia.

\section{Identificar y jerarquizar los activos}

Se cuenta con sistemas, subsistemas, componentes y partes mantenibles de los activos. A continuación, se muestra en la siguiente sección el análisis de esta parte de los equipos.

\section{Máquina pulidora-destroncadora 1}

Tiene un motor trifásico el cual es controlado por un switch reversible que le permite cambiar el sentido de giro del motor; la potencia se transmite a través de un sistema de poleas que reducen las revoluciones y hacen girar el cabezal de pulido donde se pone el resorte de amortiguación y los discos de pulido. El cabezal tiene unos ductos que permiten el paso del agua del tanque hacia los discos de pulido por medio de una llave. Este equipo es el más antiguo de todos por lo que ha sido modificado para aumentar su potencia y poder pulir superficies muy exigentes. 
Tabla 3

Jerarquización de equipos

\begin{tabular}{|c|c|c|c|c|}
\hline \multicolumn{5}{|c|}{ Formato identificación de equipo } \\
\hline Activo & Sistema & Subsistema & Componente & Parte mantenible \\
\hline \multirow[t]{11}{*}{$\begin{array}{l}\text { Destroncadora } \\
\text { pulidora }\end{array}$} & \multirow[t]{2}{*}{ Eléctrico } & Alimentación & Motor trifásico & $\begin{array}{l}\text { - Inducido } \\
\text { - Bobinas de } \\
\text { campo }\end{array}$ \\
\hline & & Control & $\begin{array}{l}\text { Switch } \\
\text { reversible }\end{array}$ & $\begin{array}{l}\text { - Platinas de } \\
\text { contacto } \\
\text { - Perilla }\end{array}$ \\
\hline & \multirow[t]{8}{*}{ Mecánico } & \multirow[t]{3}{*}{ Transmisión } & $\begin{array}{l}\text { Polea } \\
\text { conductora }\end{array}$ & - Polea de $3^{\prime \prime}$ \\
\hline & & & $\begin{array}{l}\text { Polea } \\
\text { conducida }\end{array}$ & - Polea de $8^{\prime \prime}$ \\
\hline & & & Correas & $\begin{array}{l}\text { - Correa en V } \\
\text { ref.: A-62 }\end{array}$ \\
\hline & & \multirow[t]{2}{*}{ Ejes } & Eje conductor & $\begin{array}{l}\text { - Eje en acero } \\
\text { rápido de } 1 \frac{1}{2}{ }^{\prime \prime}\end{array}$ \\
\hline & & & Eje conducido & $\begin{array}{l}\text { - Eje en acero } \\
\text { rápido de } 1 \frac{1}{21} 2^{\prime \prime} \\
\text { - Tuerca de } \\
\text { sujeción }\end{array}$ \\
\hline & & \multirow[t]{2}{*}{$\begin{array}{l}\text { Cabezal de } \\
\text { pulido }\end{array}$} & $\begin{array}{l}\text { Eje } \\
\text { transmisión }\end{array}$ & $-N / A$ \\
\hline & & & Rodamientos & - Ref. 47679 \\
\hline & & Amortiguación & $\begin{array}{l}\text { Resorte } \\
\text { amortiguador }\end{array}$ & $\begin{array}{l}\text { - Resorte de } \\
\text { acero RS-2021 }\end{array}$ \\
\hline & Hidráulico & $\begin{array}{l}\text { Tanque de } \\
\text { agua }\end{array}$ & $\begin{array}{l}\text { Llave } \\
\text { dispensadora } \\
\text { de agua }\end{array}$ & $\begin{array}{l}\text { - Embolo de } \\
\text { salida }\end{array}$ \\
\hline
\end{tabular}

Fuente: elaboración propia.

\section{Formato de ficha técnica utilizada para los equipos}

En la tabla 4 se muestra el formato de ficha técnica diseñado para los activos del proceso y en la tabla 5 la identificación del equipo que da cuenta de las partes mantenibles, cuyo stock de repuestos se requiere para las actividades de mantenimiento que serán desarrolladas en el planning que se propone en esta investigación.

\section{Análisis funcional}

En el RCM se hace énfasis en mantener la función deseada por lo que se debe conocer dicha función; una vez se conoce, se pueden inferir las fallas funcionales; es de aclarar que no todas las fallas que ocurren afectan la función (de tipo primaria); también se pueden presentar varias fallas que afecten una misma función (de tipo secundaria), como se muestra en la tabla 6.
Tabla 4

Ficha técnica de los equipos

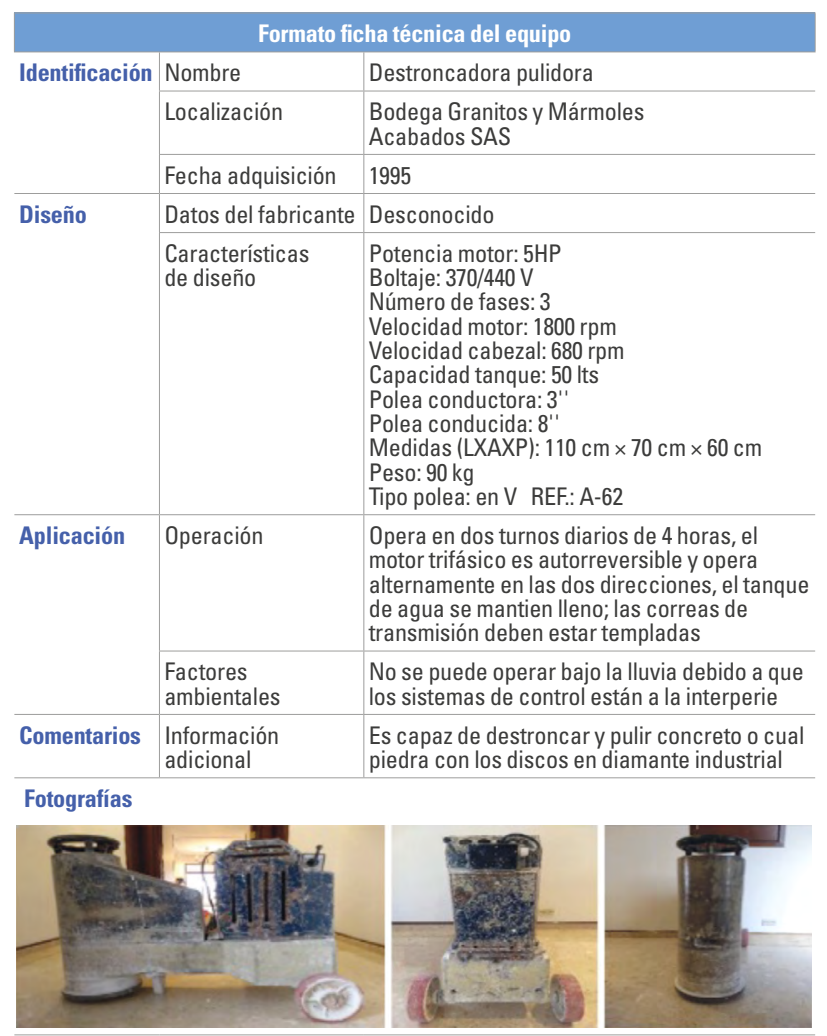

Fuente: elaboración propia.

Tabla 5

Formato de Identificación del Equipo

\begin{tabular}{|c|c|c|c|c|}
\hline \multicolumn{5}{|c|}{ Formato identificación de equipo } \\
\hline Activo & Sistema & Subsistema & Componente & $\begin{array}{l}\text { Parte } \\
\text { mantenible }\end{array}$ \\
\hline \multirow[t]{11}{*}{$\begin{array}{l}\text { Destroncadora } \\
\text { pulidora }\end{array}$} & \multirow[t]{2}{*}{ Eléctrico } & Alimentación & Motor trifásico & $\begin{array}{l}\text { - Inducido } \\
\text { - Bobinas de } \\
\text { campo }\end{array}$ \\
\hline & & Control & Switch reversible & $\begin{array}{l}\text { - Platinas de } \\
\text { contacto } \\
\text { - Perilla }\end{array}$ \\
\hline & \multirow[t]{8}{*}{ Mecánico } & \multirow[t]{3}{*}{ Transmisión } & Polea conductora & - Polea de $3^{\prime \prime}$ \\
\hline & & & Polea conducida & - Polea de 8" \\
\hline & & & Correas & $\begin{array}{l}\text { - Correa en V } \\
\text { ref.: A-62 }\end{array}$ \\
\hline & & \multirow[t]{2}{*}{ Ejes } & Eje conductor & $\begin{array}{l}\text { - Eje en acero } \\
\text { rápido de } 1 \frac{1}{21}{ }^{\prime \prime}\end{array}$ \\
\hline & & & Eje conducido & $\begin{array}{l}\text { - Eje en acero } \\
\text { rápido de } 1 \frac{112^{\prime \prime}}{} \\
\text { - Tuerca de } \\
\text { sujeción }\end{array}$ \\
\hline & & \multirow{2}{*}{$\begin{array}{l}\text { Cabezal de } \\
\text { pulido }\end{array}$} & Eje transmisión & $-N / A$ \\
\hline & & & Rodamientos & - Ref. 47679 \\
\hline & & Amortiguación & $\begin{array}{l}\text { Resorte } \\
\text { amortiguador }\end{array}$ & $\begin{array}{l}\text { - Resorte de } \\
\text { acero RS-2021 }\end{array}$ \\
\hline & Hidráulico & Tanque de agua & $\begin{array}{l}\text { Llave dispensadora } \\
\text { de agua }\end{array}$ & $\begin{array}{l}\text { - Embolo de } \\
\text { salida }\end{array}$ \\
\hline
\end{tabular}

Fuente: elaboración propia. 
Tabla 6

Análisis funcional

\begin{tabular}{|c|c|c|c|c|c|}
\hline \multirow[t]{2}{*}{ Equipo } & \multicolumn{2}{|r|}{ Función } & \multicolumn{3}{|l|}{ Falla funcional } \\
\hline & ítem & Descripción & Descripción & $\mathbf{P}$ & S \\
\hline $\begin{array}{l}\text { DES-1 } \\
\text { DES-2 } \\
\text { DES-3 }\end{array}$ & 1 & $\begin{array}{l}\text { Destroncar } 50 \mathrm{~m}^{2} / \\
\text { día de superficie } \\
\text { desbastando la } \\
\text { unión que sobresale }\end{array}$ & $\begin{array}{l}\text { 1.1 Destronque incompleto } \\
\text { 1.2 Falla en caja de transmisión, } \\
\text { cabezal no gira } \\
\text { 1.3 Falla en motor trifásico }\end{array}$ & $x$ & $\mathrm{x}$ \\
\hline $\begin{array}{l}\text { DES-2 } \\
\text { DES-3 }\end{array}$ & 2 & $\begin{array}{l}\text { Pulir } 40 \mathrm{~m}^{2} / \text { día de } \\
\text { pisos de mármol } \\
\text { dando diferentes } \\
\text { acabados }\end{array}$ & $\begin{array}{l}\text { 2.1 Pulido incompletoo no } \\
\text { uniforme } \\
\text { 2.2 Falla en caja de transmisión, } \\
\text { cabezal no gira } \\
\text { 2.3 Falla en motor trifásico }\end{array}$ & $x$ & $\mathrm{x}$ \\
\hline $\begin{array}{l}\text { BRI-1 } \\
\text { BRI-2 }\end{array}$ & 3 & $\begin{array}{l}\text { Brillar } 60 \mathrm{~m}^{2} / \text { día } \\
\text { de pisos de mármol } \\
\text { dando acabado final }\end{array}$ & $\begin{array}{l}\text { 3.1 Brillado no uniforme } \\
\text { 3.2 Falla en platinas de contacto } \\
\text { 3.3 Falla en motor trifásico }\end{array}$ & $\begin{array}{l}x \\
x\end{array}$ & $\mathrm{x}$ \\
\hline
\end{tabular}

Fuente: elaboración propia.

\section{Modos de fallas}

Se identificaron las fallas presentadas, cómo ocurrieron y las afectaciones que tuvieron; para esto se debe determinar el mecanismo y el modo de falla observado, como se muestra en la tabla 7.
Tabla 7

Modos de falla

\begin{tabular}{|c|c|c|c|}
\hline Falla funcional & Modo de falla & Mecanismo de falla & Código \\
\hline \multirow{3}{*}{$\begin{array}{l}\text { 1.1 Destronque } \\
\text { mal realizado } \\
\text { incompleto }\end{array}$} & $\begin{array}{l}\text { Falla en switch } \\
\text { reversible }\end{array}$ & $\begin{array}{l}\text { Hay energía perno } \\
\text { enciende }\end{array}$ & DES-E-CON-B \\
\hline & $\begin{array}{l}\text { Embolo } \\
\text { dispensador de } \\
\text { agua tapado }\end{array}$ & $\begin{array}{l}\text { No cae agua sobre } \\
\text { el cabezal de pulido }\end{array}$ & DES-H-TAN-K-14 \\
\hline & $\begin{array}{l}\text { Falla en resorte } \\
\text { suspensión }\end{array}$ & No hay amortiguación & DES-M-AMO-J-13 \\
\hline \multirow{3}{*}{$\begin{array}{l}\text { 1.2 Falla en caja } \\
\text { de transmisión, } \\
\text { cabezal no gira }\end{array}$} & $\begin{array}{l}\text { Correas } \\
\text { sueltas }\end{array}$ & $\begin{array}{l}\text { Baja velocidad en el } \\
\text { cabezal de pulido }\end{array}$ & DES-M-TRA-E \\
\hline & Correas rotas & $\begin{array}{l}\text { Motor arranca pero } \\
\text { no gira cabezal }\end{array}$ & DES-M-TRA-E-7 \\
\hline & $\begin{array}{l}\text { Rodamientos } \\
\text { desgastados }\end{array}$ & $\begin{array}{l}\text { Fuerte sonido } \\
\text { al arrancar }\end{array}$ & DES-M-CAB-I-12 \\
\hline
\end{tabular}

Fuente: elaboración propia.

\section{Análisis de modos y efectos de fallas (AMEF)}

Se presentaron los datos de tal manera que fuera posible evidenciar el impacto que tiene cada uno; planteando tareas para mitigarlos mediante el análisis de modos y efectos de fallas, en el cual se incluye el cálculo del RPN de acuerdo con el nivel de severidad, ocurrencia y detección, además, permite establecer las tareas de mantenimiento preventivo que requieren los equipos, como se muestra en la tabla 8 .

Tabla 8

AMEF

\begin{tabular}{|c|c|c|c|c|c|c|c|c|c|}
\hline \multicolumn{10}{|c|}{ Análisis de modo y efectos de falla } \\
\hline Falla funcional & Modo de falla & Efecto de falla potencial & S & Causas de la falla & 0 & Controles actuales & D & RPN & Acciones recomendadas \\
\hline \multirow[t]{3}{*}{$\begin{array}{l}\text { Destronque } \\
\text { o pulido mal } \\
\text { realizado } \\
\text { incompleto }\end{array}$} & $\begin{array}{l}\text { Falla en switch } \\
\text { reversible }\end{array}$ & $\begin{array}{l}\text { Pérdida total de la función; } \\
\text { afectación a la producción, } \\
\text { tiempos operacionales y } \\
\text { tiempo de entrega }\end{array}$ & 0,8 & $\begin{array}{l}\text { - Desgaste en las } \\
\text { platinas de contacto } \\
\text { - Rotura de la perilla }\end{array}$ & 3 & $\begin{array}{l}\text { - Revisar integridad } \\
\text { física del activo }\end{array}$ & 1 & 2,4 & $\begin{array}{l}\text { - Realizar pruebas pre } \\
\text { operacionales } \\
\text { - Cambio periódico del switch } \\
\text { - Inspección visual }\end{array}$ \\
\hline & $\begin{array}{l}\text { Embolo } \\
\text { dispensador de } \\
\text { agua tapado }\end{array}$ & $\begin{array}{l}\text { Proceso no deseado } \\
\text { causando reproceso; retraso } \\
\text { en tiempos de entrega, } \\
\text { pérdida de confianza }\end{array}$ & 0,4 & $\begin{array}{l}\text { - Suciedad en el } \\
\text { tanque }\end{array}$ & 2 & $\begin{array}{l}\text { - Limpiar el tanque } \\
\text { periódicamente } \\
\text { - Filtrar agua que } \\
\text { se usa }\end{array}$ & 2 & 1,6 & - Limpieza mensual del tanque \\
\hline & $\begin{array}{l}\text { Falla en resorte } \\
\text { suspensión }\end{array}$ & $\begin{array}{l}\text { Proceso no deseado } \\
\text { causando reproceso; retraso } \\
\text { en tiempos de entrega, } \\
\text { pérdida de confianza }\end{array}$ & 0,9 & $\begin{array}{l}\text { - Resorte mal ubicado } \\
\text { - Fatiga en el resorte } \\
\text { - Disco de pulido mal } \\
\quad \text { puesto }\end{array}$ & 3 & $\begin{array}{l}\text { - Revisar integridad } \\
\text { física } \\
\text { - Inspección visual } \\
\text { antes de arrancar }\end{array}$ & 1 & 2,7 & $\begin{array}{l}\text { - Inspección visual y pruebas } \\
\text { periódicas al resorte } \\
\text { - Entrenar al personal en el uso } \\
\text { correcto del equipo }\end{array}$ \\
\hline \multirow[t]{3}{*}{$\begin{array}{l}\text { Falla en caja } \\
\text { de transmisión, } \\
\text { cabezal no gira }\end{array}$} & Correas sueltas & $\begin{array}{l}\text { Reducción del torque, } \\
\text { pérdida gradual de la } \\
\text { función; retraso en tiempos } \\
\text { de entrega, pérdida } \\
\text { de confianza }\end{array}$ & 0,65 & $\begin{array}{l}\text { - Vibraciones } \\
\text { desajustan las } \\
\text { poleas } \\
\text { - Fuga de agua que } \\
\text { hace perder fricción } \\
\text { en las poleas }\end{array}$ & 4 & $\begin{array}{l}\text { - Ajuste periódico de } \\
\text { las correas } \\
\text { - Inspección visual } \\
\text { del tanque en } \\
\text { busca de fugas }\end{array}$ & 2 & 5,2 & $\begin{array}{l}\text { - Ajuste semanal de las correas } \\
\text { - Entrenar al personal } \\
\text { - Cambio periódico de las } \\
\text { correas } \\
\text { - Inspección visual del tanque } \\
\text { en busca de fugas }\end{array}$ \\
\hline & Correas rotas & $\begin{array}{l}\text { Pérdida total de la función; } \\
\text { afectación a la producción, } \\
\text { tiempos operacionales y } \\
\text { tiempos de entrega }\end{array}$ & 0,6 & $\begin{array}{l}\text { - Demasiada } \\
\text { templada las poleas } \\
\text { - Mal uso de la } \\
\text { máquina }\end{array}$ & 2 & $\begin{array}{l}\text { - Cambio de correas } \\
\text { cuando fallan }\end{array}$ & 1 & 1,2 & $\begin{array}{l}\text { - Ajuste semanal de las correas } \\
\text { - Entrenar al personal } \\
\text { - Cambio periódico de las } \\
\text { correas }\end{array}$ \\
\hline & $\begin{array}{l}\text { Rodamientos } \\
\text { desgastados }\end{array}$ & $\begin{array}{l}\text { No se puede dar inicio } \\
\text { a las labores }\end{array}$ & 0,95 & $\begin{array}{l}\text { - Fuga de agua hacia } \\
\text { caja de rodamientos }\end{array}$ & 2 & $\begin{array}{l}\text { - Inspección visual } \\
\text { del tanque en } \\
\text { busca de fugas }\end{array}$ & 3 & 5,7 & $\begin{array}{l}\text { - Lubricación periódica de la } \\
\text { caja de rodamientos }\end{array}$ \\
\hline
\end{tabular}

Fuente: elaboración propia. 


\section{Análisis RAM}

Este análisis brinda información acerca de la confiabilidad (R), la mantenibilidad (M) y la disponibilidad (A) de los equipos, lo que permite presentar un criterio para la toma de decisiones. Hay diferentes tipos de máquinas que se dividen en dos modelos, con el fin de relacionar los tiempos de intervenciones obtenidos en las fallas ocurridas en el 2018 y algunos registros que se tienen de fallas del 2017, de las cuales se calculó el tiempo entre paradas como de ocho horas de operación diaria, por 22 días al mes, por el número de meses entre cada falla; con dicha información se estimaron los tiempos promedio de falla (MTBF), tiempos medios de reparación (MTTR) y demás indicadores que son utilizados para definir las frecuencias de intervención. Estos resultados se muestran en la tabla 9.

Tabla 9.

Resultado del análisis RAM

\begin{tabular}{l|c|c|c|c|c|c|c|c} 
Modelo & MTTR & MTBF & $\begin{array}{c}\text { Tiempo prom. } \\
\text { operación }\end{array}$ & $\begin{array}{c}\text { Tiempo prom. } \\
\text { reparación }\end{array}$ & $\beta$ & $\mathbf{R}(\mathrm{t})$ & $\mathbf{A}(\mathrm{t})$ & $\mathbf{M}(\mathrm{t})$ \\
\hline $\mathrm{DES}$ & 2 & 838,58 & 1.500 & 2 & 1 & 0,17 & 0,99 & 0,61 \\
\hline $\mathrm{BRI}$ & 2 & 681,66 & 1.000 & 2,2 & 1 & 0,23 & 0,99 & 0,66 \\
\hline
\end{tabular}

Fuente: autor del proyecto.

\section{Diseño del plan de mantenimiento}

Con las acciones recomendadas, definidas anteriormente, se realizó una planificación de mantenimiento en la que se tuvieron en cuenta los recursos humanos, materiales y disposición de tiempos; este último fue de vital importancia para optimizar los recursos. Se estimaron los tiempos de intervención considerando la historia técnica y las experiencias registradas, para los equipos caso de estudio no se disponían de los antecedentes operativos ni de mantenimiento, por lo que se recurrió a la información que existe de equipos similares y a la opinión de operarios y expertos (Sierra, 2004; López, 2017), como se muestra en la tabla 10.
Tabla 10

Plan de mantenimiento preventivo

\begin{tabular}{|c|c|c|c|c|c|c|c|}
\hline \multirow{2}{*}{ Activo } & \multirow{2}{*}{ Descripción } & \multicolumn{3}{|c|}{ Recursos } & \multirow{2}{*}{ Frecuencia } & \multirow{2}{*}{$\begin{array}{l}\text { Ejecutado } \\
\text { por }\end{array}$} & \multirow{2}{*}{$\begin{array}{c}\text { Modo } \\
\text { de falla } \\
\text { asociado }\end{array}$} \\
\hline & & H & M & Tipo & & & \\
\hline DES-1 & $\begin{array}{l}\text { Cambio } \\
\text { periódico } \\
\text { del switch }\end{array}$ & $x$ & $x$ & $\begin{array}{l}\text { Herramienta } \\
\text { switch } \\
\text { reversible }\end{array}$ & Anual & Operario & $\begin{array}{l}\text { DES-E- } \\
\text { CON-B }\end{array}$ \\
\hline DES-1 & $\begin{array}{l}\text { Inspección visual } \\
\text { del switch }\end{array}$ & $x$ & & $\begin{array}{l}\text { E.P.P. } \\
\text { adecuados }\end{array}$ & Semanal & Operario & $\begin{array}{l}\text { DES-E- } \\
\text { CON-B }\end{array}$ \\
\hline DES-1 & $\begin{array}{l}\text { Limpieza del } \\
\text { tanque }\end{array}$ & $x$ & $x$ & Grata cepillos & Mensual & Operario & $\begin{array}{l}\text { DES-H- } \\
\text { TAN-K-14 }\end{array}$ \\
\hline DES-1 & $\begin{array}{l}\text { Realizar pruebas } \\
\text { de carga al } \\
\text { resorte }\end{array}$ & $x$ & $x$ & \begin{tabular}{|l|} 
Pesos de \\
pruebay y \\
calibrador
\end{tabular} & Mensual & Operario & $\begin{array}{l}\text { DES-M- } \\
\text { AM0-J-13 }\end{array}$ \\
\hline DES-1 & $\begin{array}{l}\text { Ajuste de } \\
\text { correas }\end{array}$ & $x$ & $x$ & Herramienta & Semanal & Operario & $\begin{array}{l}\text { DES-M- } \\
\text { TRA-E-7 }\end{array}$ \\
\hline DES-1 & $\begin{array}{l}\text { Cambio periódico } \\
\text { correas }\end{array}$ & $x$ & $x$ & $\begin{array}{l}\text { Herramienta } \\
\text { Correa A-62 } \\
\end{array}$ & Anual & Operario & $\begin{array}{l}\text { DES-M- } \\
\text { TRA-E-7 }\end{array}$ \\
\hline DES-1 & $\begin{array}{l}\text { Lubricación caja } \\
\text { de rodamientos }\end{array}$ & $x$ & $x$ & $\begin{array}{l}\text { Herramienta } \\
\text { Grasa de litio }\end{array}$ & Semestral & Operario & $\begin{array}{l}\text { DES-M- } \\
\text { CAB-I-12 }\end{array}$ \\
\hline
\end{tabular}

Fuente: elaboración propia.

\section{Programar las actividades de mantenimiento preventivo}

Se crearon rutas de mantenimiento donde se especificaban las tareas que deben realizarse semanal, mensual, semestral o anualmente mediante un formato de ruta de mantenimiento, como se muestra en la tabla 11.

Tabla 11

Formato de ruta de trabajo para mantenimiento preventivo

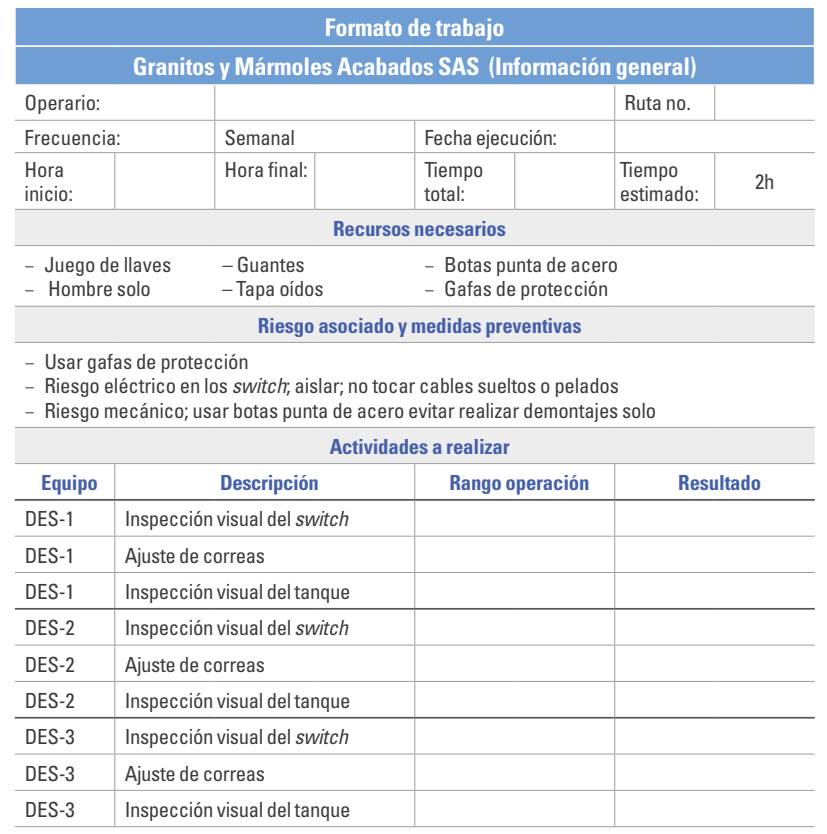

Fuente: elaboración propia. 


\section{Planificación de paradas (implementación)}

Con los aportes brindados por los colaboradores y las personas que manejan los equipos, se estableció el mejor momento para ejecutar las rutas, teniendo en cuenta que siempre se debe fijar un responsable para su ejecución, así como asegurarse que el operario no tiene más tareas por realizar en el momento, de acuerdo con lo siguiente:

Las rutas semanales consisten en actividades de inspección y ajuste que no necesitan mucho tiempo, por lo que pueden ser ejecutadas por cualquier operario. Deben ser realizadas al inicio de la semana, reprogramándolas (de ser necesario, el operario que esté programado ese día es quien la realiza).

Las rutas mensuales tienen actividades un poco más complejas de realizar porque necesitan el desmonte del tanque, por lo que, para garantizar la disponibilidad de los equipos, se programó la ejecución de estas en el transcurso de una semana y deben ser llevadas a cabo por el mismo operario quien interviene un equipo por día; en el planning de mantenimiento se indican con la letra $\mathrm{M}$ en la semana donde se debe cumplir.

Las rutas semestrales se programaron para los meses de febrero y agosto debido a que son las épocas cuando baja el flujo de trabajo, esto da dos semanas de tiempo, de forma tal que se cruzan con una ruta mensual para aprovechar el desmonte realizado; esta requiere al operario y al ayudante para hacer más eficaz y seguro el desarrollo de las actividades. Se muestran en el planning con una letra $\mathrm{S}$ en las semanas donde se ha planeado.

Las rutas anuales se proyectaron para agosto; son intervenciones a los dispositivos de control y las realizadas por terceros, por lo tanto, no se cuenta con ellos por un día cada uno y se deben desplazar al taller; se le dio un mes para la ejecución y cierre de la ruta. A continuación, se muestra el planning de mantenimiento desde el inicio de la implementación del plan y para el 2019 (González, 2016; Hernández, 2006), como se muestra en la tabla 12.

Tabla 12

Planning de mantenimiento

\begin{tabular}{|c|c|c|c|c|c|c|c|c|c|c|c|c|c|c|c|c|c|c|c|c|c|c|c|c|c|}
\hline \multirow{2}{*}{ Descripción } & \multirow{2}{*}{$\begin{array}{c}\text { Mes } \\
\text { Estado }\end{array}$} & \multicolumn{4}{|c|}{ Enero } & \multicolumn{4}{|c|}{ Febrero } & \multicolumn{4}{|c|}{ Marzo } & \multicolumn{4}{|c|}{ Abril } & \multicolumn{4}{|c|}{ Mayo } & \multicolumn{4}{|c|}{ Junio } \\
\hline & & 1 & 2 & 3 & 4 & 1 & 2 & 3 & 4 & 1 & 2 & 3 & 4 & 1 & 2 & 3 & 4 & 1 & 2 & 3 & 4 & 1 & 2 & 3 & 4 \\
\hline \multirow{2}{*}{ Destroncadora 1} & Ejecutado & & & $x$ & & & $x$ & $x$ & & & & $\mathrm{x}$ & & & & $x$ & & & & & & & & & \\
\hline & Planeado & & & $M$ & & & $S$ & M & & & & $\mathrm{M}$ & & & & $M$ & & & & $M$ & & & & $M$ & \\
\hline Destroncadora 2 & Planeado & & & M & & & & $\mathrm{M} / \mathrm{S}$ & & & & $\mathrm{M}$ & & & & $\mathrm{M}$ & & & & M & & & & $\mathrm{M}$ & \\
\hline \multirow[t]{2}{*}{ Destroncadora 3} & Ejecutado & & & $x$ & & & $x$ & $x$ & & & & $\mathrm{X}$ & & & & $x$ & & & & & & & & & \\
\hline & Planeado & & & M & & & S & M & & & & $\mathrm{M}$ & & & & $M$ & & & & M & & & & M & \\
\hline Pulidora brilladora industrial 1 & Planeado & & & $M$ & & & & $\mathrm{M} / \mathrm{S}$ & & & & M & & & & $M$ & & & & $M$ & & & & $M$ & \\
\hline \multirow[t]{2}{*}{ Pulidora brilladora industrial 1} & Ejecutado & & & $x$ & & & $x$ & $x$ & & & & $x$ & & & & $x$ & & & & & & & & & \\
\hline & Planeado & & & M & & & $S$ & M & & & & $\mathrm{M}$ & & & & $M$ & & & & M & & & & $M$ & \\
\hline \multirow{2}{*}{ Descripción } & Mes & \multicolumn{4}{|c|}{ Julio } & \multicolumn{4}{|c|}{ Agosto } & \multicolumn{4}{|c|}{ Septiembre } & \multicolumn{4}{|c|}{ Octubre } & \multicolumn{4}{|c|}{ Noviembre } & \multicolumn{4}{|c|}{ Diciembre } \\
\hline & Estado & 1 & 2 & 3 & 4 & 1 & 2 & 3 & 4 & 1 & 2 & 3 & 4 & 1 & 2 & 3 & 4 & 1 & 2 & 3 & 4 & 1 & 2 & 3 & 4 \\
\hline \multirow[t]{2}{*}{ Destroncadora 1} & Ejecutado & & & & & & & & & & & & & & & & & & & & & & & & \\
\hline & Planeado & & & M & & A & $S$ & M & & & & M & & & & M & & & & M & & & & $M$ & \\
\hline Destroncadora 2 & Planeado & & & M & & $A$ & & $\mathrm{M} / \mathrm{S}$ & & & & $\mathrm{M}$ & & & & $M$ & & & & $M$ & & & & $M$ & \\
\hline \multirow[t]{2}{*}{ Destroncadora 3} & Ejecutado & & & & & & & & & & & & & & & & & & & & & & & & \\
\hline & Planeado & & & $M$ & & & S & M & A & & & M & & & & M & & & & M & & & & $M$ & \\
\hline Pulidora brilladora industrial 1 & Ejecutado & & & & & & & & & & & & & & & & & & & & & & & & \\
\hline & Planeado & & & $M$ & & $A$ & & $\mathrm{M} / \mathrm{S}$ & & & & M & & & & $M$ & & & & $M$ & & & & $M$ & \\
\hline Pulidora brilladora industrial 1 & Ejecutado & & & & & & & & & & & & & & & & & & & & & & & & \\
\hline & Planeado & & & $M$ & & & $S$ & M & $A$ & & & M & & & & M & & & & $M$ & & & & $M$ & \\
\hline Rutas semanales: se ejecut & n los lunes & ma & teng & es fe & tivo) & & & Rutas & 0 & tra & (con & רdic & or & a & la & eme & qu & e ej & outa & & & & & & \\
\hline Rutas mensuales: se indica & con una M & las & man & que & e ej & utar & & Rutas & anua & es: s & ejec & tará & en a & sto & e in & ica c & n un & & & & K: se & & 19 & se ej & cutó \\
\hline
\end{tabular}

Fuente: elaboración propia. 


\section{Análisis financiero}

Con esta herramienta se analizaron los costos asociados a la producción y al desarrollo de las actividades necesarias para el cumplimiento de las funciones en el transcurso de la implementación del plan, también se tuvo en cuenta el lucro cesante debido a las fallas y la tasa de inversión representativa de la implementación. Para realizar este estudio se evaluaron los eventos transcurridos desde octubre del 2018 hasta abril del 2019 (García et al., 2003).

\section{Inversión asociada a la implementación del plan de mantenimiento}

Este proyecto fue realizado por el estudiante de Ingeniería Mecánica Jhon Sebastián Rocha, quien se encargó de recolectar la información, generar el plan y posteriormente seguir su ejecución; ha prestado sus servicios en dicha empresa a cambio del desarrollo de su opción de grado por lo que no representó ningún costo; en cuanto a consumibles se usaron una resma de papel, bolígrafos y marcadores Sharpie, por un valor de $\$ 33.000$ pesos. Además, se adquirieron los repuestos necesarios para dar cumplimiento al plan durante un año, como se muestra en la tabla 13.

Tabla 13

Stock de repuestos para un año

\begin{tabular}{|c|c|c|c|c|}
\hline Descripción & Cant. & \begin{tabular}{|c} 
Valor \\
unitario
\end{tabular} & $\begin{array}{l}\text { Valor } \\
\text { total }\end{array}$ & Aplicación \\
\hline $\begin{array}{l}\text { Grasa blanca } \\
\text { de litio } 3 \text { en uno }\end{array}$ & 1 & $\$ 27.000$ & $\$ 27.000$ & Lubricar los cabezales de pulido \\
\hline $\begin{array}{l}\text { Traba rosca } \\
\text { loctite } 277\end{array}$ & 1 & $\$ 120.000$ & $\$ 120.000$ & $\begin{array}{l}\text { Ajustar la tuerca de sujeción de la polea } \\
\text { conducida al cabezal de pulido }\end{array}$ \\
\hline Silicona roja & 2 & $\$ 8.000$ & $\$ 16.000$ & Sellar las uniones en los cabezales de pulido \\
\hline Correa ref.: A-60 & 6 & $\$ 8.000$ & $\$ 48.000$ & $\begin{array}{l}\text { Transmitir la potencia del motor } \\
\text { a cabezal de pulido }\end{array}$ \\
\hline Correa ref.: A-62 & 6 & $\$ 8.000$ & $\$ 48.000$ & $\begin{array}{l}\text { Transmitir la potencia del motor } \\
\text { a cabezal de pulido }\end{array}$ \\
\hline Correa ref.: A-66 & 6 & $\$ 8.000$ & $\$ 48.000$ & $\begin{array}{l}\text { Transmitir la potencia del motor } \\
\text { a cabezal de pulido }\end{array}$ \\
\hline $\begin{array}{l}\text { Switch reversible } \\
\text { para motor trifásico }\end{array}$ & 3 & $\$ 75.000$ & $\$ 225.000$ & Invierte la dirección de giro del motor \\
\hline Switch de seguridad & 2 & $\$ 56.000$ & $\$ 112.000$ & $\begin{array}{l}\text { Evita que la máquina funcione sin } \\
\text { la presencia del operario }\end{array}$ \\
\hline Platinas de contacto & 4 & $\$ 15.000$ & $\$ 60.000$ & Cierran el circuito en el motor bifásico \\
\hline Cuña de transmisión & 2 & $\$ 30.000$ & $\$ 60.000$ & $\begin{array}{l}\text { Transmitir la potencia del motor } \\
\text { a cabezal de pulido }\end{array}$ \\
\hline Capacitor & 2 & $\$ 38.000$ & $\$ 76.000$ & $\begin{array}{l}\text { Regula la corriente antes de ingresar } \\
\text { al motor bifásico }\end{array}$ \\
\hline \multicolumn{3}{|l|}{ Total } & $\$ 840.000$ & \\
\hline
\end{tabular}

Fuente: elaboración propia.
Tiempo y costos invertidos en las rutas que se han diseñado en el plan de mantenimiento

Estas rutas serán ejecutadas por el operario, por lo que representará una inversión el tiempo que use para dichas intervenciones; el sueldo del operario es $\$ 1.500 .000$, asumiendo que labora 192 horas mensuales cada hora cuesta $\$ 7.812,5$. Para la fecha en la cual se hizo el estudio fueron ejecutadas, como se muestra en la tabla 14:

- 28 rutas semanales, las cuales necesitan dos horas para ser ejecutadas, lo que se traduce en 56 horas fuera de servicio, además, estas no han generado costos ni requerido elementos del stock, ya que consisten en inspecciones visuales.

- Ocho rutas mensuales que representan 28 horas fuera de servicio a la fecha en las cuales no se ha empleado ningún repuesto.

- Una ruta semestral que representó siete horas de afectación donde se usaron tres correas Ref.: A-60, A-62, A-66, dos porta platinas para las brilladoras industriales y grasa de litio para lubricar los cabezales y rodamientos.

- Las rutas anuales aún no habían sido ejecutadas, pero se estima el tiempo que se necesitan para su ejecución.

Tabla 14

Tiempos y costos invertidos en las rutas

\begin{tabular}{l|c|c|c|c|c|c} 
Frecuencia & $\begin{array}{c}\text { Tiempo } \\
\text { por ruta }\end{array}$ & $\begin{array}{c}\text { Rutas } \\
\text { ejecutadas }\end{array}$ & $\begin{array}{c}\text { Turas } \\
\text { planeadas }\end{array}$ & $\begin{array}{c}\text { Tiempo } \\
\text { ejecutado }\end{array}$ & $\begin{array}{c}\text { Tiempo } \\
\text { planeado }\end{array}$ & $\begin{array}{c}\text { Total } \\
\text { repuestos }\end{array}$ \\
\hline Semanal & 2 & 32 & 48 & 64 & 96 & N/A \\
\hline Mensual & 4 & 8 & 12 & 32 & 48 & N/A \\
\hline Semestral & 8 & 1 & 2 & 8 & 16 & $\$ 116.000$ \\
\hline Anual & 4 & N/A & 1 & N/A & 4 & N/A \\
\hline Total tiempo invertido planeado & & & & 164 \\
\hline Total tiempo invertido a la fecha \\
Total repuestos \\
Flujo cesante debido a la ejecución
\end{tabular}

Fuente: elaboración propia.

La inversión total del plan de mantenimiento para la fecha del estudio corresponde a lo indicado en la tabla 15 mostrada a continuación: 
Tabla 15

Inversión total del plan de mantenimiento a la fecha del estudio

\begin{tabular}{l|r}
\multicolumn{1}{c|}{ Inversión } & \multicolumn{1}{c}{ Valor } \\
\hline Recurso humano e insumos & $\$ 33.000$ \\
\hline Stock de repuestos para un año & $\$ 840.000$ \\
\hline Ejecución de las rutas & $\$ 718.750$ \\
\hline Total inversión & $\$ 1.591 .750$ \\
\hline
\end{tabular}

Fuente: elaboración propia.

Para realizar el análisis financiero hay que tener en cuenta los costos totales del plan de mantenimiento para la fecha del estudio, los cuales se muestran a continuación en la figura 18.

Tabla 16

Costos totales del plan de mantenimiento a la fecha del estudio

\begin{tabular}{l|r}
\multicolumn{1}{c|}{ Costos } & \multicolumn{1}{c}{ Valor } \\
\hline Costos de mantenimiento correctivo & $\$ 520.000$ \\
\hline Costos de mantenimiento terceros & $\$ 380.000$ \\
\hline Stock de repuestos, insumos y recurso humano & $\$ 873.000$ \\
\hline Lucro cesante & $\$ 827.309$ \\
\hline Ejecución de las rutas & $\$ 718.750$ \\
\hline Total costos & $\$ 3.319 .059$
\end{tabular}

Fuente: elaboración propia.

\section{Cálculo del ROI}

En este caso el beneficio de implementar el plan de mantenimiento es la ganancia dejada de obtener debido a dicha implementación, así como todos los costos asociados al mantenimiento realizado (Oreda, 2002), por lo que:

$$
\begin{aligned}
& \begin{aligned}
\text { Beneficio }= & \left(\text { costos totales } \times \text { ganancia esperada }{ }^{\star}\right) \\
& + \text { costos de } \text { mtto }
\end{aligned} \\
& \text { Beneficio }=(\$ 2 .) 939.059 * 0,4+\$ 520.000+\$ 718.750
\end{aligned}
$$

$$
\text { Beneficio }=\$ 2.414 .376
$$

* La ganancia esperada que plantea

el dueño de la empresa es del $40 \%$.

La inversión necesaria para la implementación es \$1.591.750 (véase ecuación 1):

$$
\begin{aligned}
& R O I=\frac{(\text { Beneficio }- \text { Inversión })}{\text { Inversión }} \\
& R O I=(\$ 2) 414.376-\$ 1.591 .750 \$ 1.591 .750 \\
& R O I=0,51
\end{aligned}
$$

Esto indica que el beneficio obtenido con respecto a la inversión es del $51 \%$, lo que significa que cada $\$ 1.000$ invertido en el plan representara $\$ 1.500$ de ganancia al final del ejercicio; además supera la ganancia esperada por el gerente. Esta inversión se realizó al inicio de la implementación y se puede estimar el tiempo en que será recuperada por medio de una regla de tres:

$$
\begin{aligned}
& \text { Tiempo }=12 \text { meses } * \$ 1.591-750 \$ 2.414 .376 \\
& \text { Tiempo }=7,91 \text { meses }
\end{aligned}
$$

Por lo que se recupera en ocho meses.

\section{Cálculo del VPN}

Con el fin de verificar la viabilidad del proyecto se complementa con el análisis del VPN, teniendo en cuenta que el flujo de caja serán los beneficios obtenidos en el año, pues todos tienen el mismo Índice de Precios al Consumidor (IPC) que fue del 3,18\% para el 2018.

Despejando el valor presente de la fórmula del interés compuesto se tiene en la ecuación 2 :

$$
V P N=\frac{V F}{(1+i)^{n}}
$$

Donde:

VPN: valor presente neto

VF: valor futuro

$i$ : tasa de interés

$\mathrm{n}$ : número de periodos entre el valor presente y el valor futuro

Reemplazando los valores se tiene:

$V P N=-\$ 1 \cdot 591 \cdot 750+\$ 2 \cdot 414 \cdot 376(1+0,0318)^{1}$

$V P N=\$ 748.215$ 
Ya que este valor es positivo se demuestra que fue conveniente realizar la inversión, como se muestra en la figura 3.

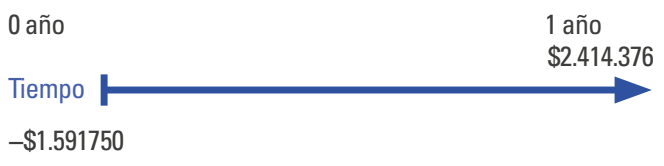

Figura 3. Flujo de caja para la inversión del plan de mantenimiento Fuente: elaboración propia.

\section{Cálculo de la TIR}

La TIR es el valor que debe tomar $i$ para que el VPN sea cero por lo que se utiliza la ecuación 3 :

$$
0=-\$ 1.591750+\$ 2.414 .376+T I R
$$

\section{Despejando}

$$
\begin{aligned}
& T I R=\$ 2.414 .376 \$ 1 \cdot 591 \cdot 750-1 \\
& T I R=0,52
\end{aligned}
$$

Además, la TIR resulta ser un porcentaje muy alto, lo que confirma lo analizado con el ROI (Norma Técnica Colombiana, 2018; Oreda, 2002).

\section{Conclusiones}

Por medio del análisis de criticidad, se identificaron y listaron los equipos críticos en la operación, a los cuales se les generaron las fichas técnicas y hojas de vida, llevando un registro de los mismos.

Los formatos de fichas técnicas, identificación de equipos y jerarquización, permitieron visualizar el stock de repuestos necesarios para cada equipo, de esta manera se tiene un mayor control sobre los recursos invertidos en estos rubros.

La implementación de la metodología RCM en las pequeñas empresas, como se aprecia en este caso estudio, brinda herramientas necesarias para organizar la información y tener mayor control de los activos.
El plan de mantenimiento que se creó, está basado en los modos de falla identificados en el AMEF y no necesariamente en los equipos; esto brinda un amplia perspectiva de cómo fallan los mismos y permite generar acciones correctivas.

Las actividades de mantenimiento correctivo requieren una inversión mayor y no fueron incluidas en este estudio; sin embargo, las rutinas de inspección que serán realizadas en forma preventiva permitirán tomar acciones para llevarlas a cabo sin que esto afecte la calidad y la productividad.

Con el análisis RAM se establecen las bases para el cálculo de los indicadores de fiabilidad, los cuales se ajustarán según mejore la calidad de los datos, permitiendo tomar decisiones más acertadas.

El plan propuesto ha permitido favorablemente organizar las actividades que los operarios venían realizando de forma aleatoria, por lo que se han optimizado los tiempos generando buenas prácticas de mantenimiento.

Con el análisis financiero del ROI, VPN y TIR se demostró que es rentable la implementación del plan de mantenimiento, ya que estos indicadores mostraron óptimos resultados. Además se evidencia que la inversión retorna en un corto plazo. La rentabilidad del proyecto se debe a que, con acciones sencillas, se mitigan fallas que pueden resultar críticas dañando las operaciones, afectando la calidad y cumplimiento con los clientes.

Declaración de conflicto de interés: Los autores no manifiestan conflictos de interés institucionales ni personales.

\section{Referencias}

Da Costa, M. (2011). Aplicación del mantenimiento centrado en la confiabilidad a motores a gas de dos tiempos en pozos de alta producción. [Tesis para optar el título de Ingeniero Mecánico, Pontificia Universidad Católica del Perú]. http://tesis.pucp.edu.pe/repositorio/ handle/20.500.12404/567

García, S. (2003). Organización y gestión integral de mantenimiento: Manual práctico para la implantación de 
sistemas de gestión avanzados de mantenimiento industrial. Ediciones Díaz de Santos.

Gómez, I. D. (2006). Introducción al Mantenimiento Estratégico. Universidad Libre.

González, D.A. (2016). Creación de planes de mantenimiento para nuevos equipos de Nestlé Purina Fábrica La Encrucijada. Universidad Simón Bolívar.

Hernández, R., Fernández, C., \& Baptista, P. (2006). Metodología de la investigación. McGraw Hill.

Huerta, R. (2007). El análisis de criticidad, una metodología para mejorar la confiabilidad operacional. Docplayer. https://docplayer.es/14621533-El-analisis-de-critici dad-una-metodologia-para-mejorar-la-confiabilidadoperacional-1-definiciones-importantes-regresar.html

López, I.E. (2017). Mantenimiento FMEA del sistema de alimentación del motor Wartsila 50DF-12V. [Tesis para optar el título como Marino, Universidad del País Vasco]. https://addi.ehu.es/bitstream/handle/10810/22769/1\% C3\%B1aki\%20Elexpuru\%20TFG\%20Septiembre17. pdf? sequence $=2 \&$ isAllowed $=y$

Lucia, J. M. (1990). Criterios para la información de la gestión del mantenimiento. Revista Mantenimiento, 1.https:// vdocuments.es/criterios-para-la-informacin-de-la-ges tin-del-criterios-para-la-informacin-de.html

Mora, L. (2019). Mantenimiento. Planeación, ejecución y control. Alfaomega.

Monsalve, M.M., \& Tena, E.A. (2018). Plan de mantenimiento para la flota de vehículos de la empresa $\mathrm{Na}$ vitrans SAS. [Tesis para optar el título de Ingeniero Mecánico, Universidad Libre de Colombia]. https://re pository.unilibre.edu.co/bitstream/handle/10901/ $11600 /$ correciones $\% 20$ finales $\% 20$ agosto $\% 20$ tena. pdf? sequence=1\&isAllowed $=y$

Moubray, J. (2000). RCM2. Reliability Centered Maintenance. Butterworth-Heinemann.
Norma Técnica Colombiana. (2008). Referencias Bibliográficas. Contenido, Forma y Estructura. NTC 5313. ICONTEC. https://www.politecnicojic.edu.co/images/downloads/ biblioteca/guias/NTC5613.pdf

Norma Técnica Colombiana. (2008). Documentación. Presentación de tesis, trabajos de grado y otros trabajos de investigación. NTC 1486. ICONTEC. http://www.unipamplona. edu.co/unipamplona/portallG/home_15/recursos/01_ general/09062014/n_icontec.pdf

Oreda. (2002) Offshore Reliability Data Handbook. Oreda. https://www.nri.ac.ir/Portals/0/images/Technology/ OandM/document/OREDA.pdf

Páramo, S. (2016). Análisis para la implementación de un plan de mantenimiento basado en confiabilidad para la maquinaria en la línea de pulido de vidrio de la empresa Vitrinas Páramo Ortega. [Tesis para optar el título de Ingeniero Mecánico, Universidad Libre de Colombia]. https://repository.unilibre.edu.co/handle/10901/10387

Salguero, M. F. (2010). Diseño e implementación de un sistema de mantenimiento estratégico aplicando las filosofías rcm y fmea a las máquinas y herramientas de la Empresa WEATHERFORD SOUTH AMERICA INC, Base1, Francisco De Orellana [Escuela Politécnica del Ejército]. http://repo sitorio.espe.edu.ec/xmlui/handle/21000/2418

Sierra, G.A. (2004). Programa de mantenimiento preventivo para la empresa Metalmecánica Industrias AMV SA. [Tesis para optar el título de Ingeniero Mecánico, Universidad Industrial de Santander]. http://tangara.uis.edu.co/ biblioweb/tesis/2004/112490.pdf

Troffé, M. (s.f). Análisis ISO 14224 /OREDA. Relación con RCMFMEA. http://www.mantenimientomundial.com/notas/ 0605MarioTroffelSO14224.pdf

Urrego, J (2017). Elaboración de un plan de mantenimiento preventivo para equipos de la línea de perforación de la empresa cimentaciones de Colombia LTDA. Universidad Santo Tomás. https://repository.usta.edu.co/bitstream/ handle/11634/11685/2017juanurrego.pdf?sequence=1 